\title{
A universal difference method for time-space fractional Black-Scholes equation
}

\author{
Yang Xiaozhong, Wu Lifei*, Sun Shuzhen and Zhang Xue
}

"Correspondence:

wulf@ncepu.edu.cn

School of Mathematics and Physics,

North China Electric Power

University, Beijing, 102206, P.R. China

\begin{abstract}
The fractional Black-Scholes (B-S) equation is an important mathematical model in finance engineering, and the study of its numerical methods has very significant practical applications. This paper constructs a new kind of universal difference method to solve the time-space fractional B-S equation. The universal difference method is analyzed to be stable, convergent, and uniquely solvable. Furthermore, it is proved that with numerical experiments the universal difference method is valid and efficient for solving the time-space fractional B-S equation. At the same time, numerical experiments indicate that the time-space fractional $\mathrm{B}-\mathrm{S}$ equation is more consistent with the actual financial market.
\end{abstract}

Keywords: time-space fractional Black-Scholes equation; universal difference method; stability; convergence; numerical experiments

\section{Introduction}

The Black-Scholes (B-S) equation is an important mathematical model in option pricing theory of finance engineering. In the financial market, the extensive application of B-S option pricing model has been driven by the rapid development of the financial derivatives market $[1,2]$. However, as we know the classical B-S model was established under some strict assumptions. According to the research on the stock market, the hypothesis of the traditional B-S equation is so idealistic that it is not completely consistent with the actual stock movement. Some extensions of the B-S model are obtained by weakening these assumptions, such as the fractional B-S model [3-8], the B-S model with transactions costs [9], the jump-diffusion model [10] etc.

During the past few decades, many important phenomena in electromagnetics, acoustics, viscoelasticity, and material science could be well described by fractional differential equations [11-13]. This is due to the fact that a realistic model of a physical phenomenon has a dependence not only on the time instant, but also the previous time history can be successfully described by using fractional calculus [14-18].

In recent years, progress has been made in the study of the fractional B-S equation. Wyss first deduced the fractional B-S equation with a time fractional derivative to price European call option [3]. Later, Jumarie applied the fractional Taylor formula to derive the fractional B-S equation based on the classical B-S equation [4]. Jumarie promoted the previous work and gave an optimal fractional Merton's portfolio, which has wider applications in the actual financial market [5]. Cartea and del-Castillo-Negrete obtained several frac-

(c) 2016 Xiaozhong et al. This article is distributed under the terms of the Creative Commons Attribution 4.0 International License (http://creativecommons.org/licenses/by/4.0/), which permits unrestricted use, distribution, and reproduction in any medium, provided you give appropriate credit to the original author(s) and the source, provide a link to the Creative Commons license, and indicate if changes were made. 
tional diffusion models of option prices in markets with jumps and priced barrier option using the fractional partial differential equation [6].

The time-space fractional option pricing model - the time-space fractional B-S equation $[3,5]$ takes the form

$$
P_{t}^{(\alpha)}(S, t)=\left(\frac{r}{\Gamma(2-\alpha)} P-r S^{\alpha} P_{S}^{(\alpha)}\right) t^{1-\alpha}-\frac{\Gamma^{3}(1+\alpha)}{\Gamma(1+2 \alpha)} \Gamma^{2}(2-\alpha) \sigma^{2} S^{2 \alpha} P_{S}^{(2 \alpha)} .
$$

Here $t>0,0<\alpha \leq 1$ and $P(S, t)$ is the European call option price at asset price $S$ and time $t, r$ is the risk free interest rate. $\sigma$ represents the volatility of underlying asset, and $\alpha$ denotes the fractional order. The fractional derivatives $P_{t}^{(\alpha)}(S, t), P_{S}^{(\alpha)}$, and $P_{S}^{(2 \alpha)}$ are RiemannLiouville time fractional derivatives.

There exists no perfect analytic solution of the time-space fractional B-S equation, so it is important to study its numerical solutions. At present, there are a few achievements on the numerical methods for solving the fractional B-S equation [19-24]. Kumar et al. provided analytic solution of the fractional B-S option pricing equation by homotopy perturbation method with coupling of the Laplace transform [19]. In 2014, they also presented a numerical algorithm for the time fractional B-S equation with boundary condition by homotopy perturbation method and homotopy analysis method [20]. Song and Wang employed implicit finite difference method to solve the time fractional B-S equation together with the conditions satisfied by the standard put options [22]. Yang et al. proposed an ImplicitExplicit and Explicit-Implicit difference scheme for the time fractional B-S equation [23]. However, up to now there is no research on the numerical methods of the time-space fractional B-S equation.

Based on the existing problems, this paper mainly studies the numerical methods of the time-space fractional B-S option pricing model in the actual financial market. We combine the call options to construct the universal difference scheme for solving the time-space fractional B-S equation. The existence and uniqueness of a numerical solution, computational stability, and convergence of the universal difference scheme are analyzed. Finally, numerical experiments demonstrate the effectiveness of the universal difference scheme for solving the time-space fractional B-S equation.

\section{Universal difference scheme of time-space fractional B-S equation}

\subsection{Time-space fractional B-S equation}

In order to get the value of a European call option, equation (1) must be integrated with boundary conditions for numerical solutions. There are three boundary conditions:

(1) $P(S, T)=\max \{S-K, 0\}$. This condition is quite clear, the profit and loss when the option expires is its price. Here, $K$ is the exercise price.

(2) $S \rightarrow \infty, P(S, t) \sim S-K e^{-r(T-t)}$. This condition means when $S$ is sufficiently great, the option price is close to $S-K e^{-r(T-t)}$. $T$ is the due date of the options.

(3) $P(0, t)=0$. This condition means when $S$ is zero, the option price is approximant to zero.

Therefore, the European call option pricing is to solve the following equation:

$$
\left\{\begin{array}{l}
P_{t}^{(\alpha)}=\left(\frac{r}{\Gamma(2-\alpha)} P-r S^{\alpha} P_{S}^{(\alpha)}\right) t^{1-\alpha}-\frac{\Gamma^{3}(1+\alpha)}{\Gamma(1+2 \alpha)} \Gamma^{2}(2-\alpha) \sigma^{2} S^{2 \alpha} P_{S}^{(2 \alpha)}, \\
P(S, T)=\max (S-K, 0) .
\end{array}\right.
$$

Equation (2) is an anti-variable coefficient parabolic equation. 
Boundary conditions:

$$
P(0, t)=0, \quad \lim _{S \rightarrow+\infty} P(S, t)=S-K e^{-r(T-t)} .
$$

Solution region:

$$
\Sigma=\{0 \leq S \leq \infty, 0 \leq t \leq T\}
$$

In order to construct difference scheme, we make the following coordinate transform [5]: $S=e^{x}, t=T-\tau, P(S, t)=e^{-r \tau} V(x, \tau)$. Equation (2) converses into the following parabolic equation:

$$
\left\{\begin{array}{l}
V_{\tau}^{(\alpha)}(x, \tau)-\left[\gamma(\alpha) \frac{\Gamma(1-\alpha)}{\Gamma(1-2 \alpha)} \sigma^{2}+r(T-\tau)^{1-\alpha}\right] \tau^{1-\alpha}(T-\tau)^{\alpha-1} V_{x}(x, \tau) \\
\quad-\gamma(\alpha) \sigma^{2} \tau^{1-\alpha}(T-\tau)^{\alpha-1} V_{x x}(x, \tau)=0 \\
V(x, 0)=\max \left(e^{x}-K, 0\right)
\end{array}\right.
$$

Here, $\gamma(\alpha)=\frac{\Gamma^{3}(1+\alpha) \Gamma^{2}(2-\alpha)}{\Gamma(1+2 \alpha)}$.

The solution region converses into

$$
\Sigma_{0}=\{-\infty \leq x<+\infty, 0 \leq \tau \leq T\}
$$

In the theory, the price of the underlying asset will not always appear to be zero or infinity. Therefore, we provide a small enough number $M^{-}$as the lower boundary and a large enough number $M^{+}$as the upper boundary in actual computation.

Therefore, the solution region converses into a finite domain:

$$
\Sigma_{1}=\left\{M^{-} \leq x<M^{+}, 0 \leq \tau \leq T\right\}
$$

At the same time, the boundary conditions converse into

$$
V\left(M^{+}, \tau\right)=e^{M^{+}+r \tau}-K, \quad V\left(M^{-}, \tau\right)=0 .
$$

\subsection{Universal difference scheme}

$h, k$ are defined, respectively, as a spatial step and a time step, here $h=\frac{M^{+}-M^{-}}{M}, k=\frac{T}{N}$.

$M$ and $N$ are positive integers.

$$
\begin{cases}x_{i}=M^{-}+(i-1) h, & i=1,2, \ldots, M+1 \\ \tau_{n}=(n-1) k, & n=1,2, \ldots, N+1 .\end{cases}
$$

The approximate value of equation (3) in the point $\left(x_{i}, \tau_{n}\right)$ is defined as $V_{i}^{n}$.

In order to construct the universal difference scheme ( $\theta$-difference scheme), we shall introduce the classic explicit scheme and implicit scheme of equation (3).

The classic explicit scheme of equation (3):

$$
\begin{aligned}
\frac{\partial^{\alpha} V\left(x_{i}, \tau_{n+1}\right)}{\partial \tau^{\alpha}}= & {\left[a b+r(T-n k+k)^{1-\alpha}\right](n k-k)^{1-\alpha}(T-n k+k)^{\alpha-1} \frac{V_{i+1}^{n}-V_{i-1}^{n}}{2 h} } \\
& +a(n k-k)^{1-\alpha}(T-n k+k)^{\alpha-1} \frac{V_{i+1}^{n}-2 V_{i}^{n}+V_{i-1}^{n}}{h^{2}} .
\end{aligned}
$$


The classic implicit scheme of equation (3):

$$
\begin{aligned}
\frac{\partial^{\alpha} V\left(x_{i}, \tau_{n+1}\right)}{\partial \tau^{\alpha}}= & {\left[a b+r(T-n k)^{1-\alpha}\right](n k)^{1-\alpha}(T-n k)^{\alpha-1} \frac{V_{i+1}^{n+1}-V_{i-1}^{n+1}}{2 h} } \\
& +a(n k)^{1-\alpha}(T-n k)^{\alpha-1} \frac{V_{i+1}^{n+1}-2 V_{i}^{n+1}+V_{i-1}^{n+1}}{h^{2}}
\end{aligned}
$$

Here, $a=\gamma(\alpha) \sigma^{2}, b=\frac{\Gamma(1-\alpha)}{\Gamma(1-2 \alpha)}, n=1,2, \ldots, N, i=1,2, \ldots, M$.

Then we assume a parameter $\theta(0 \leq \theta \leq 1)$, and let $(1-\theta)$ multiply equation (4), $\theta$ multiply equation (5), then add up their results,

$$
\begin{aligned}
& \frac{\partial^{\alpha} V\left(x_{i}, \tau_{n+1}\right)}{\partial \tau^{\alpha}} \\
& =(1-\theta)\left[\left(a b+r(T-n k+k)^{1-\alpha}\right)(n k-k)^{1-\alpha}(T-n k+k)^{\alpha-1} \frac{V_{i+1}^{n}-V_{i-1}^{n}}{2 h}\right. \\
& \left.\quad+a(n k-k)^{1-\alpha}(T-n k+k)^{\alpha-1} \frac{V_{i+1}^{n}-2 V_{i}^{n}+V_{i-1}^{n}}{h^{2}}\right] \\
& \quad+\theta\left[\left(a b+r(T-n k)^{1-\alpha}\right)(n k)^{1-\alpha}(T-n k)^{\alpha-1} \frac{V_{i+1}^{n+1}-V_{i-1}^{n+1}}{2 h}\right. \\
& \left.\quad+a(n k)^{1-\alpha}(T-n k)^{\alpha-1} \frac{V_{i+1}^{n+1}-2 V_{i}^{n+1}+V_{i-1}^{n+1}}{h^{2}}\right] .
\end{aligned}
$$

The discrete scheme of time fractional derivative is as follows:

$$
\frac{\partial^{\alpha} V\left(x_{i}, \tau_{n+1}\right)}{\partial \tau^{\alpha}}=\frac{k^{-\alpha}}{\Gamma(2-\alpha)} \sum_{j=1}^{n}\left[V\left(x_{i}, \tau_{n+2-j}\right)-V\left(x_{i}, \tau_{n+1-j}\right)\right]\left[j^{1-\alpha}-(j-1)^{1-\alpha}\right] .
$$

Ignoring the errors, we can get equation (6):

$$
\begin{aligned}
& \frac{k^{-\alpha}}{\Gamma(2-\alpha)} \sum_{j=1}^{n}\left[V\left(x_{i}, \tau_{n+2-j}\right)-V\left(x_{i}, \tau_{n+1-j}\right)\right]\left[j^{1-\alpha}-(j-1)^{1-\alpha}\right] \\
& \quad=(1-\theta)\left[\left(a b+r(T-n k+k)^{1-\alpha}\right)(n k-k)^{1-\alpha}(T-n k+k)^{\alpha-1} \frac{V_{i+1}^{n}-V_{i-1}^{n}}{2 h}\right. \\
& \left.\quad+a(n k-k)^{1-\alpha}(T-n k+k)^{\alpha-1} \frac{V_{i+1}^{n}-2 V_{i}^{n}+V_{i-1}^{n}}{h^{2}}\right] \\
& \quad+\theta\left[\left(a b+r(T-n k)^{1-\alpha}\right)(n k)^{1-\alpha}(T-n k)^{\alpha-1} \frac{V_{i+1}^{n+1}-V_{i-1}^{n+1}}{2 h}\right. \\
& \left.\quad+a(n k)^{1-\alpha}(T-n k)^{\alpha-1} \frac{V_{i+1}^{n+1}-2 V_{i}^{n+1}+V_{i-1}^{n+1}}{h^{2}}\right] .
\end{aligned}
$$

Equation (6) is the universal difference scheme for equation (3), it can be written as follows:

$$
\begin{aligned}
V_{i}^{n+1}- & V_{i}^{n}+\sum_{j=2}^{n}\left[V\left(x_{i}, \tau_{n+2-j}\right)-V\left(x_{i}, \tau_{n+1-j}\right)\right] l_{j} \\
= & m_{1}\left[(1-\theta)\left(a b g_{n}+r q_{n}\right)\left(V_{i+1}^{n}-V_{i-1}^{n}\right)+\theta\left(a b g_{n+1}+r q_{n+1}\right)\left(V_{i+1}^{n+1}-V_{i-1}^{n+1}\right)\right] \\
& +m_{2}\left[(1-\theta) g_{n}\left(V_{i+1}^{n}-2 V_{i}^{n}+V_{i-1}^{n}\right)+\theta g_{n+1}\left(V_{i+1}^{n+1}-2 V_{i}^{n+1}+V_{i-1}^{n+1}\right)\right] .
\end{aligned}
$$


Sorting out the last equation, then we can get the following equation:

$$
\begin{aligned}
{[-} & \left.m_{1}\left(a b g_{n+1}+r q_{n+1}\right)-m_{2} g_{n+1}\right] \theta V_{i+1}^{n+1}+\left(1+2 \theta m_{2} g_{n+1}\right) V_{i}^{n+1} \\
& +\left[m_{1}\left(a b g_{n+1}+r q_{n+1}\right)-m_{2} g_{n+1}\right] \theta V_{i-1}^{n+1} \\
= & (1-\theta)\left[m_{1}\left(a b g_{n}+r q_{n}\right)+m_{2} g_{n}\right] V_{i+1}^{n}+\left[1-2 m_{2} g_{n}(1-\theta)\right] V_{i}^{n} \\
& +(1-\theta)\left[-m_{1}\left(a b g_{n}+r q_{n}\right)+m_{2} g_{n}\right] V_{i-1}^{n}-\left(2^{1-\alpha}-1\right) V_{i}^{n}+\sum_{j=2}^{n-1} d_{j} V_{i}^{n+1-j}+l_{n} V_{i}^{1} \\
= & (1-\theta)\left[m_{1}\left(a b g_{n}+r q_{n}\right)+m_{2} g_{n}\right] V_{i+1}^{n}+\left[-2 m_{2} g_{n}(1-\theta)\right] V_{i}^{n} \\
& +(1-\theta)\left[-m_{1}\left(a b g_{n}+r q_{n}\right)+m_{2} g_{n}\right] V_{i-1}^{n}+\sum_{j=1}^{n-1} d_{j} V_{i}^{n+1-j}+l_{n} V_{i}^{1} .
\end{aligned}
$$

The matrix form of universal difference scheme is as follows:

$$
\left\{\begin{array}{l}
G_{1} V^{n+1}=\left(G_{2}+I d_{1}\right) V^{n}+B, \\
B=\sum_{j=2}^{n-1} d_{j} V^{n+1-j}+l_{n} V^{1}+C, \\
C=\left(a_{n}^{\prime} V_{1}^{n}-a_{n+1} V_{1}^{n+1}, 0, \ldots, 0, c_{n}^{\prime} V_{M+1}^{n}-c_{n+1} V_{M+1}^{n+1}\right)^{\prime},
\end{array} \quad n=1,2,3, \ldots, N .\right.
$$

The matrix (8) can be written as follows:

$$
\begin{aligned}
& \left(\begin{array}{ccccc}
b_{n+1} & c_{n+1} & & & \\
a_{n+1} & b_{n+1} & c_{n+1} & & \\
& \ddots & \ddots & \ddots & \\
& & a_{n+1} & b_{n+1} & c_{n+1} \\
& & & a_{n+1} & b_{n+1}
\end{array}\right)\left(\begin{array}{c}
V_{2}^{n+1} \\
V_{3}^{n+1} \\
\vdots \\
V_{M-1}^{n+1} \\
V_{M}^{n+1}
\end{array}\right) \\
& =\left(\begin{array}{ccccc}
b_{n}^{\prime} & c_{n}^{\prime} & & \\
a_{n}^{\prime} & b_{n}^{\prime} & c_{n}^{\prime} & & \\
& \ddots & \ddots & \ddots & \\
& & a_{n}^{\prime} & b_{n}^{\prime} & c_{n}^{\prime} \\
& & & a_{n}^{\prime} & b_{n}^{\prime}
\end{array}\right)\left(\begin{array}{c}
V_{2}^{n} \\
V_{3}^{n} \\
\vdots \\
V_{M-1}^{n} \\
V_{M}^{n}
\end{array}\right)+\sum_{j=1}^{n-1} d_{j}\left(\begin{array}{c}
V_{2}^{n+1-j} \\
V_{3}^{n+1-j} \\
\vdots \\
V_{M-1}^{n+1-j} \\
V_{M}^{n+1-j}
\end{array}\right) \\
& +l_{n}\left(\begin{array}{c}
V_{2}^{1} \\
V_{3}^{1} \\
\vdots \\
V_{M-1}^{1} \\
V_{M}^{1}
\end{array}\right)+\left(\begin{array}{c}
a_{n}^{\prime} V_{1}^{n}-a_{n+1} V_{1}^{n+1} \\
0 \\
\vdots \\
0 \\
c_{n}^{\prime} V_{M+1}^{n}-c_{n+1} V_{M+1}^{n+1}
\end{array}\right) \\
& \sum_{j=1}^{n-1} d_{j}\left(\begin{array}{c}
V_{2}^{n+1-j} \\
V_{3}^{n+1-j} \\
\vdots \\
V_{M-1-j}^{n+1-j} \\
V_{M}^{n+1-j}
\end{array}\right)=d_{1}\left(\begin{array}{c}
V_{2}^{n} \\
V_{3}^{n} \\
\vdots \\
V_{M-1}^{n} \\
V_{M}^{n}
\end{array}\right)+d_{2}\left(\begin{array}{c}
V_{2}^{n-1} \\
V_{3}^{n-1} \\
\vdots \\
V_{M-1}^{n-1} \\
V_{M}^{n-1}
\end{array}\right)+d_{3}\left(\begin{array}{c}
V_{2}^{n-2} \\
V_{3}^{n-2} \\
\vdots \\
V_{M-1}^{n-2} \\
V_{M}^{n-2}
\end{array}\right)+\cdots+d_{n-1}\left(\begin{array}{c}
V_{2}^{2} \\
V_{3}^{2} \\
\vdots \\
V_{M-1}^{2} \\
V_{M}^{2}
\end{array}\right) .
\end{aligned}
$$


Here, $m_{1}=\Gamma(2-\alpha) k^{\alpha} / 2 h, m_{2}=\gamma(\alpha) \sigma^{2} \Gamma(2-\alpha) k^{\alpha} / h^{2}$,

$$
\begin{aligned}
& l_{j}=j^{1-\alpha}-(j-1)^{1-\alpha}, \quad d_{j}=2 j^{1-\alpha}-(j+1)^{1-\alpha}-(j-1)^{1-\alpha}, \quad j=1,2, \ldots, n, \\
& g_{n}=(n k-k)^{1-\alpha}(T-n k+k)^{\alpha-1}, \quad q_{n}=(n k-k)^{1-\alpha}, \\
& a_{n+1}=\theta\left[m_{1}\left(a b g_{n+1}+r q_{n+1}\right)-m_{2} g_{n+1}\right], \\
& b_{n+1}=1+2 \theta m_{2} g_{n+1}, \quad c_{n+1}=\theta\left[-m_{1}\left(a b g_{n+1}+r q_{n+1}\right)-m_{2} g_{n+1}\right], \\
& a_{n}^{\prime}=(1-\theta)\left[-m_{1}\left(a b g_{n}+r q_{n}\right)+m_{2} g_{n}\right], \\
& b_{n}^{\prime}=-2 m_{2} g_{n}(1-\theta), \quad c_{n}^{\prime}=(1-\theta)\left[m_{1}\left(a b g_{n}+r q_{n}\right)+m_{2} g_{n}\right] .
\end{aligned}
$$

\section{The theoretical analysis of universal difference scheme for the time-space fractional B-S equation}

\subsection{Existence and uniqueness of the universal difference scheme solution}

For $G_{1}$, there are $a_{n+1}<0, c_{n+1}<0, b_{n+1}>0$ and $b_{n+1}-\left|a_{n+1}+c_{n+1}\right|=1$, so the matrix $G_{1}$ is a diagonally dominant matrix. In other words, the coefficient matrix $G_{1}$ is an invertible matrix.

For $G_{2}$, there are $a_{n}^{\prime}>0, c_{n}^{\prime}>0, b_{n}^{\prime}<0$ and $\left|b_{n}^{\prime}\right|-\left|a_{n}^{\prime}+c_{n}^{\prime}\right|=0$, so the matrix $G_{2}+I d_{1}$ is a diagonally dominant matrix. In other words, the coefficient matrix $G_{2}+I d_{1}$ is an invertible matrix. Therefore, the universal difference scheme (6) has an unique solution.

Theorem 1 The universal difference scheme (6) for the time-space fractional B-S equation is uniquely solvable.

\subsection{Stability and convergence of universal difference scheme}

Lemma 1 The following equations hold [14]:

$$
0<d_{n}<\cdots<d_{2}<d_{1}<1, \quad d_{j}=l_{j}-l_{j+1}, \quad \sum_{j=1}^{n-1} d_{j}=1-l_{n}, \quad l_{1}=1 .
$$

Lemma 2 Assume that $\tilde{V}_{i}^{n}$ is the approximate solution of universal difference scheme (6), and $\varepsilon_{i}^{n}=\tilde{V}_{i}^{n}-V_{i}^{n}, E^{n}=\left(\varepsilon_{1}^{n}, \varepsilon_{2}^{n}, \ldots, \varepsilon_{m-1}^{n}\right)$, then when $\frac{1}{2} \leq \theta \leq 1$ for any $1 \leq n \leq N+1$, one will set $\left\|E^{n}\right\|_{\infty} \leq\left\|E^{1}\right\|_{\infty}$; when $0 \leq \theta<\frac{1}{2}$ and $\frac{a \Gamma(2-\alpha) k^{\alpha}}{h^{2}} N^{\alpha-1}<1$, one will set $\left\|E^{n}\right\|_{\infty} \leq\left\|E^{1}\right\|_{\infty}$.

Proof Applying mathematical induction.

When $n=1$, one will set

$$
c_{2} \varepsilon_{i+1}^{2}+b_{2} \varepsilon_{i}^{2}+a_{2} \varepsilon_{i-1}^{2}=\varepsilon_{i}^{1}
$$

When $n>1$, one will set

$$
c_{n+1} \varepsilon_{i+1}^{n+1}+b_{n+1} \varepsilon_{i}^{n+1}+a_{n+1} \varepsilon_{i-1}^{n+1}=c_{n}^{\prime} \varepsilon_{i+1}^{n}+b_{n}^{\prime} \varepsilon_{i}^{n}+a_{n}^{\prime} \varepsilon_{i-1}^{n}+\sum_{j=1}^{n-1} d_{j} \varepsilon_{i}^{n-j}+l_{n} \varepsilon_{i}^{1} .
$$

Define $\left|\varepsilon_{l}^{2}\right|=\max _{2 \leq i \leq M}\left|\varepsilon_{i}^{2}\right|$, then one will set

$$
\left|\varepsilon_{l}^{2}\right| \leq c_{2}\left|\varepsilon_{l+1}^{2}\right|+b_{2}\left|\varepsilon_{l}^{2}\right|+a_{2}\left|\varepsilon_{l-1}^{2}\right| \leq\left|c_{2} \varepsilon_{l+1}^{2}+b_{2} \varepsilon_{l}^{2}+a_{2} \varepsilon_{l-1}^{2}\right|=\left|\varepsilon_{i}^{1}\right|=\left\|E^{1}\right\|_{\infty} .
$$

Assuming $n \leq s$, we will have $\left\|E^{n}\right\|_{\infty} \leq\left\|E^{1}\right\|_{\infty}$. 
When $n=s+1$, assume $\left|\varepsilon_{l}^{s+1}\right|=\max _{2 \leq i \leq M}\left|\varepsilon_{i}^{s+1}\right|$, then one will set

$$
\begin{aligned}
\left|\varepsilon_{l}^{s+1}\right| \leq & c_{s+1}\left|\varepsilon_{l+1}^{s+1}\right|+b_{s+1}\left|\varepsilon_{l}^{s+1}\right|+a_{s+1}\left|\varepsilon_{l-1}^{s+1}\right| \\
\leq & \left|c_{s+1} \varepsilon_{l+1}^{s+1}+b_{s+1} \varepsilon_{l}^{s+1}+a_{s+1} \varepsilon_{l-1}^{s+1}\right| \\
= & \left|c_{s}^{\prime} \varepsilon_{i+1}^{s}+b_{s}^{\prime} \varepsilon_{i}^{s}+a_{s}^{\prime} \varepsilon_{i-1}^{s}+\sum_{j=1}^{s-1} d_{j} \varepsilon_{i}^{s+1-j}+l_{s} \varepsilon_{i}^{1}\right| \\
\leq & c_{s}^{\prime}\left\|E^{s}\right\|_{\infty}+b_{s}^{\prime}\left\|E^{s}\right\|_{\infty}+a_{s}^{\prime}\left\|E^{s}\right\|_{\infty}+d_{1}\left\|E^{s}\right\|_{\infty}+d_{2}\left\|E^{s}\right\|_{\infty}+\cdots \\
& +d_{s-1}\left\|E^{s}\right\|_{\infty}+l_{s}\left\|E^{1}\right\|_{\infty} \\
= & d_{1}\left\|E^{s}\right\|_{\infty}+d_{2}\left\|E^{s}\right\|_{\infty}+\cdots+d_{s-1}\left\|E^{s}\right\|_{\infty}+l_{s}\left\|E^{1}\right\|_{\infty} \\
\leq & \left(d_{1}+d_{2}+\cdots+d_{s-1}+l_{s}\right)\left\|E^{1}\right\|_{\infty}=\left\|E^{1}\right\|_{\infty} .
\end{aligned}
$$

Obviously, we have the conclusion $\left\|E^{s+1}\right\|_{\infty} \leq\left\|E^{1}\right\|_{\infty}$.

Therefore, we can obtain the following theorem.

Theorem 2 When $\frac{1}{2} \leq \theta \leq 1$, the universal difference scheme (6) for the time-space fractional $B-S$ equation is stable; when $0 \leq \theta<\frac{1}{2}$ and the inequality $\frac{a \Gamma(2-\alpha) k^{\alpha}}{h^{2}} N^{\alpha-1}<1$ holds, the universal difference scheme (6) for the time-space fractional B-S equation is stable.

Lemma 3 Assuming $V\left(x_{i}, \tau_{n}\right)$ is the exact solution of the differential equation on the mesh point $\left(x_{i}, \tau_{n}\right)$, and defining $e_{i}^{n}=V\left(x_{i}, \tau_{n}\right)-V_{i}^{n}, e^{1}=0, e^{n}=\left(e_{1}^{n}, e_{2}^{n}, \ldots, e_{m-1}^{n}\right),\left\|e^{n}\right\|_{\infty}=$ $\max _{1 \leq i \leq m-1}\left|e_{i}^{n}\right|$, here, $n=1,2, \ldots, N$, then when $\frac{1}{2} \leq \theta \leq 1$, one will set $\left\|e^{n}\right\|_{\infty} \leq l_{n-1}^{-1} H\left(\tau^{1-\alpha}+\right.$ $\left.\tau^{\alpha} h^{2}\right)$; when $0 \leq \theta<\frac{1}{2}$ and the inequality $\frac{a \Gamma(2-\alpha) k^{\alpha}}{h^{2}} N^{\alpha-1}<1$ holds, one will set $\left\|e^{n}\right\|_{\infty} \leq$ $l_{n-1}^{-1} H\left(\tau^{1-\alpha}+\tau^{\alpha} h^{2}\right)$. Here, $H$ is a constant.

Proof We will apply mathematical induction. Substitute $V_{i}^{n}=V\left(x_{i}, \tau_{n}\right)-e_{i}^{n}$ into the difference scheme.

When $n=1$, one will set

$$
c_{2} e_{i+1}^{2}+b_{2} e_{i}^{2}+a_{2} e_{i-1}^{2}=R_{i}^{1}
$$

When $n>1$, one will set

$$
c_{n+1} e_{i+1}^{n+1}+b_{n+1} e_{i}^{n+1}+a_{n+1} e_{i-1}^{n+1}=c_{n}^{\prime} e_{i+1}^{n}+b_{n}^{\prime} e_{i}^{n}+a_{n}^{\prime} e_{i-1}^{n}+\sum_{j=1}^{n-1} d_{j} e_{i}^{n-j}+R_{i}^{n+1}
$$

Here, $\left\|R_{i}^{n+1}\right\| \leq H\left(\tau^{1+\alpha}+\tau^{\alpha} h^{2}\right), H$ is a constant, $n=1,2, \ldots, N$.

When $n=1$, assuming $\left|e_{l}^{2}\right|=\max _{2 \leq i \leq M}\left|e_{i}^{2}\right|$ the relation is as follows:

$$
\begin{aligned}
\left\|e^{2}\right\|_{\infty} & =\left|e_{l}^{2}\right| \leq c_{2}\left|e_{l+1}^{2}\right|+b_{2}\left|e_{l}^{2}\right|+a_{2}\left|e_{l-1}^{2}\right| \\
& \leq\left|c_{2} e_{l+1}^{2}+b_{2} e_{l}^{2}+a_{2} e_{l-1}^{2}\right| \\
& =\left|R_{l}^{1}\right| \leq H\left(\tau^{1+\alpha}+\tau^{\alpha} h^{2}\right) \\
& =l_{1}^{-1} H\left(\tau^{1+\alpha}+\tau^{\alpha} h^{2}\right) .
\end{aligned}
$$


Assuming $k \leq s$, we will have $\left\|e^{k+1}\right\|_{\infty} \leq l_{k}^{-1} H\left(\tau^{1+\alpha}+\tau^{\alpha} h^{2}\right)$. We already have $l_{j}^{-1} \leq l_{k}^{-1}$, $j=1,2, \ldots, k$. Then, when $k=s+1$ and assuming $\left|e_{l}^{s+1}\right|=\max _{2 \leq i \leq M}\left|e_{i}^{s+1}\right|$, we obtain the following result:

$$
\begin{aligned}
\left\|e^{s+1}\right\|_{\infty}= & \left|e_{l}^{s+1}\right| \leq c_{s+1}\left|e_{l+1}^{s+1}\right|+b_{s+1}\left|e_{l}^{s+1}\right|+a_{s+1}\left|e_{l-1}^{s+1}\right| \\
\leq & \left|c_{s+1} e_{l+1}^{s+1}+b_{s+1} e_{l}^{s+1}+a_{s+1} e_{l-1}^{s+1}\right| \\
= & \left|c_{s}^{\prime} e_{l+1}^{s}+b_{s}^{\prime} e_{l}^{s}+a_{s}^{\prime} e_{l-1}^{s}+\sum_{j=1}^{s-1} d_{j} e_{i}^{s+1-j}+R_{l}^{s+1}\right| \\
\leq & c_{s}^{\prime}\left\|e^{s}\right\|_{\infty}+b_{s}^{\prime}\left\|e^{s}\right\|_{\infty}+a_{s}^{\prime}\left\|e^{s}\right\|_{\infty}+d_{1}\left\|e^{s}\right\|_{\infty}+d_{2}\left\|e^{s-1}\right\|_{\infty}+\cdots \\
& +d_{s-1}\left\|e^{2}\right\|_{\infty}+H\left(\tau^{1+\alpha}+\tau^{\alpha} h^{2}\right) \\
= & d_{1}\left\|e^{s}\right\|_{\infty}+d_{2}\left\|e^{s-1}\right\|_{\infty}+\cdots+d_{s-1}\left\|e^{2}\right\|_{\infty}+H\left(\tau^{1+\alpha}+\tau^{\alpha} h^{2}\right) \\
\leq & \left(d_{1} l_{s-1}^{-1}+d_{2} l_{s-2}^{-1}+\cdots+d_{s-1} l_{1}^{-1}+1\right) H\left(\tau^{1+\alpha}+\tau^{\alpha} h^{2}\right) \\
\leq & \left(\sum_{j=1}^{s-1} l_{s}^{-1} d_{j}+1\right) H\left(\tau^{1+\alpha}+\tau^{\alpha} h^{2}\right) \\
= & l_{s}^{-1}\left(\sum_{j=1}^{s-1} d_{j}+l_{s}\right) H\left(\tau^{1+\alpha}+\tau^{\alpha} h^{2}\right) \\
= & l_{s}^{-1} H\left(\tau^{1+\alpha}+\tau^{\alpha} h^{2}\right) .
\end{aligned}
$$

Because

$$
\lim _{n \rightarrow \infty} \frac{l_{n}^{-1}}{n^{\alpha}}=\lim _{n \rightarrow \infty} \frac{n^{-\alpha}}{n^{1-\alpha}-(n-1)^{1-\alpha}}=\lim _{n \rightarrow \infty} \frac{n^{-1}}{1-\left(1-\frac{1}{n}\right)^{1-\alpha}}=\frac{1}{1-\alpha},
$$

there is a constant $c>0$, by which we can obtain

$$
\left\|e^{n}\right\|_{\infty} \leq n^{\alpha} c\left(\tau^{1+\alpha}+\tau^{\alpha} h^{2}\right)=(n \tau)^{\alpha} c\left(\tau+h^{2}\right), \quad n=1,2, \ldots, N .
$$

We know $n \tau \leq T$, which is a limit number, then we can get the conclusion as follows:

$$
\left|V\left(x_{i}, \tau_{n}\right)-V_{i}^{n}\right| \leq \tilde{c}\left(\tau+h^{2}\right), \quad i=2,3, \ldots, M, n=1,2, \ldots, N .
$$

Here, $\tilde{c}=T^{\alpha} c$.

Theorem 3 When $\frac{1}{2} \leq \theta \leq 1$, the universal difference scheme (6) for the time-space fractional B-S equation is convergent; when $0 \leq \theta<\frac{1}{2}$ and the inequality $\frac{a \Gamma(2-\alpha) k^{\alpha}}{h^{2}} N^{\alpha-1}<1$ holds, the universal difference scheme (6) for the time-space fractional B-S equation is convergent, and the degree of convergence is first-order time and second-order space.

\section{Numerical examples}

Using a Pentium (R) Dual Core CPU 3.00 GHz, we will experiment by utilizing the universal difference scheme in the Matlab 7.0 environment. In order to compare with the integer order B-S equation, we use the universal difference scheme to calculate the price of a European call option. 
Table 1 The price of a European call option $(\alpha=5 / 7, M=200, N=40)$

\begin{tabular}{llllll}
\hline Time (T/month) & $\mathbf{3}$ & $\mathbf{6}$ & $\mathbf{9}$ & $\mathbf{1 2}$ & The stability \\
\hline $\boldsymbol{\theta}=1$ & 45.0580 & 45.3705 & 45.7238 & 46.1680 & stable \\
$\theta=2 / 3$ & 45.2422 & 45.8177 & 46.5242 & 47.7146 & stable \\
$\theta=1 / 2$ & 45.3345 & 46.0423 & 46.9278 & 48.4993 & stable \\
$\theta=1 / 3$ & 45.4269 & 46.2676 & 47.3336 & 49.2917 & unstable \\
\hline
\end{tabular}

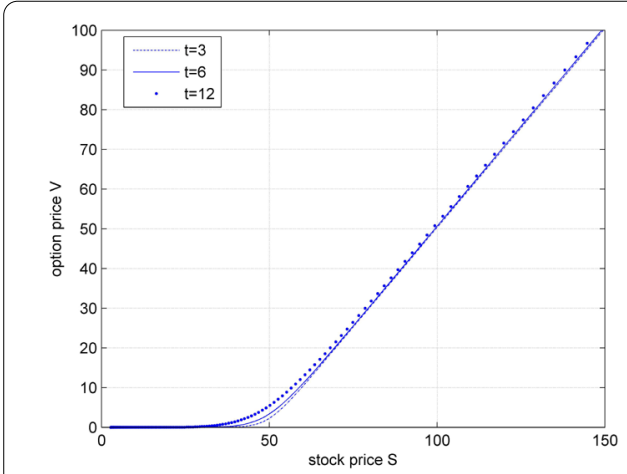

(a) $\theta=1$

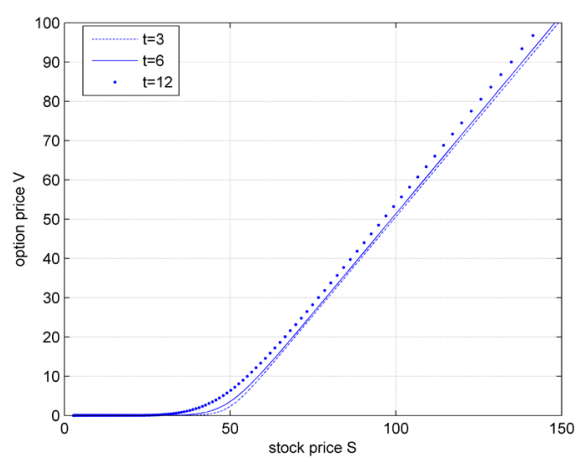

(c) $\theta=1 / 2$

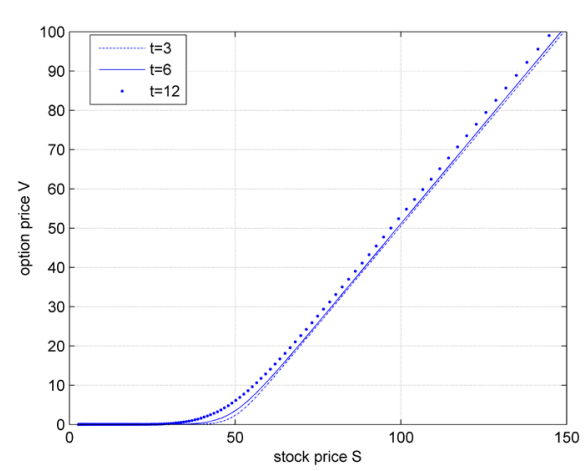

(b) $\theta=2 / 3$

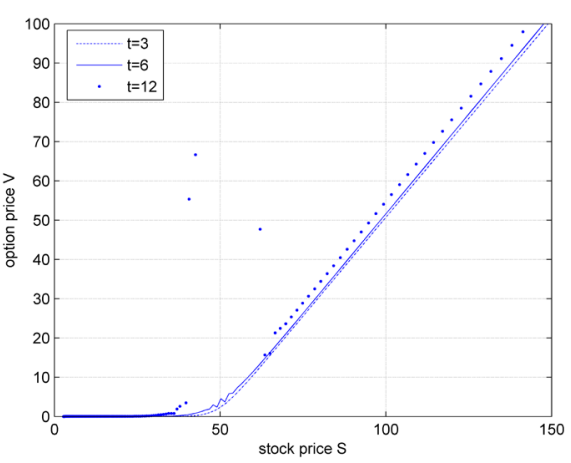

(d) $\theta=1 / 3$

Figure 1 The price of a European call option $(\alpha=5 / 7, M=200, N=40)$.

Example Supposing a European call option, whose maturity is 3, 6, 12 months, the current price of the stock is $97 \$$, the strike price is $50 \$$, the risk free nominal interest rate is $1 \%$, and the stock's volatility is $20 \%$.

Solution The parameters are

$$
S=97, \quad K=50, \quad T=1, \quad r=0.01, \quad \sigma=0.2, \quad M^{+}=\ln 300, \quad M^{-}=1 .
$$

Then we take different spatial steps (see case I and case II) and temporal steps to compute the numerical solutions.

Case I:

$$
\begin{aligned}
& \alpha=5 / 7, \quad \theta=1,2 / 3,1 / 2,1 / 3, \quad M=200, \quad N=40, \quad k=0.025, \quad h=0.0235, \\
& \frac{a \Gamma(2-\alpha) k^{\alpha}}{h^{2}} N^{\alpha-1}=1.13>1 .
\end{aligned}
$$

We get the results in Table 1 and Figure 1. 
Table 2 The price of a European call option $(\alpha=5 / 7, M=200, N=120)$

\begin{tabular}{llllll}
\hline Time (T/month) & $\mathbf{3}$ & $\mathbf{6}$ & $\mathbf{9}$ & $\mathbf{1 2}$ & The stability \\
\hline$\theta=1$ & 45.0578 & 45.3698 & 45.7219 & 46.2439 & stable \\
$\theta=2 / 3$ & 45.2641 & 45.8436 & 46.5591 & 47.9096 & stable \\
$\theta=1 / 2$ & 45.3675 & 46.0816 & 46.9812 & 48.7626 & stable \\
$\theta=1 / 3$ & 45.4709 & 46.3204 & 47.4058 & 49.6250 & stable \\
\hline
\end{tabular}

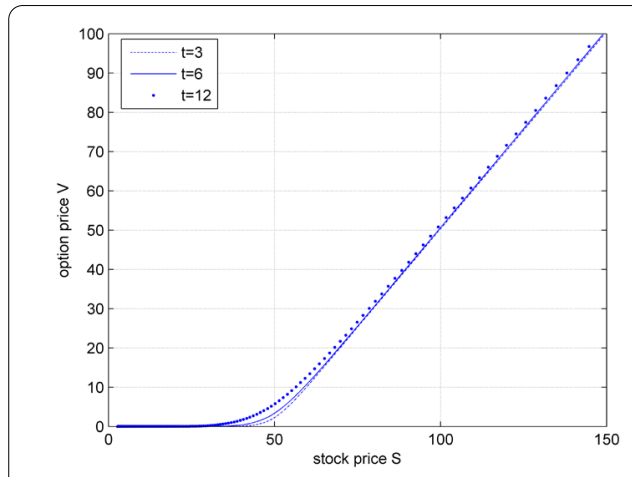

(a) $\theta=1$

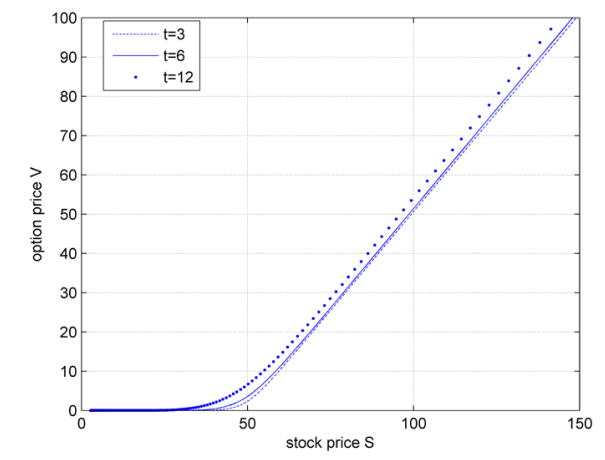

(c) $\theta=1 / 2$

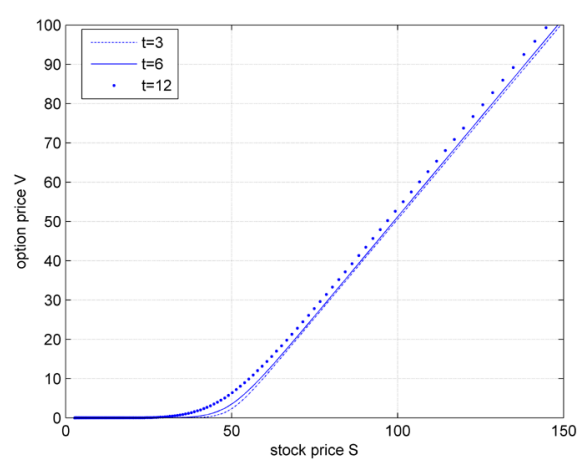

(b) $\theta=2 / 3$

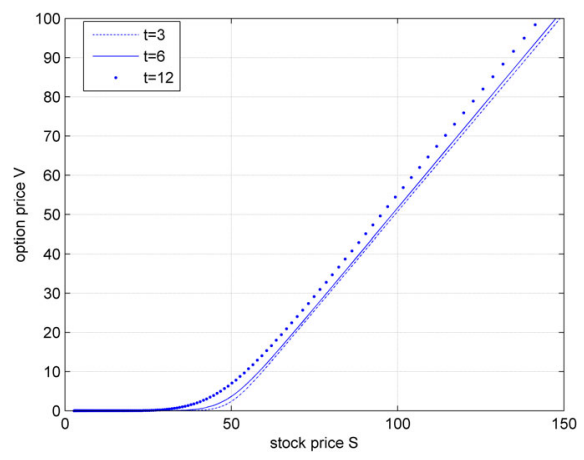

(d) $\theta=1 / 3$

Figure 2 The price of a European call option $(\alpha=5 / 7, M=200, N=120)$.

From the numerical solution in Table 1 and Figure 1 , we can see that when $\theta=1 / 3$, the universal difference scheme does not satisfy the stability condition, so the calculation is unstable and the numerical solutions have few references; when selecting $\theta=1 / 2,2 / 3,1$, the universal difference scheme satisfies the stability condition, so the calculation is stable.

Case II:

$$
\begin{aligned}
& \alpha=5 / 7, \quad \theta=1,2 / 3,1 / 2,1 / 3, \quad M=200, \quad N=120, \quad k=0.0083, \quad h=0.0235, \\
& \frac{a \Gamma(2-\alpha) k^{\alpha}}{h^{2}} N^{\alpha-1}=0.37<1 .
\end{aligned}
$$

We get the results in Table 2 and Figure 2.

According to the given date in Table 2 and Figure 2 and the theoretical analysis, we can see that when selecting $\theta=1,2 / 3,1 / 2,1 / 3$, the universal difference scheme satisfies the stability condition, so the calculation is stable. The results above indicate that the universal difference scheme (6) for the time-space fractional B-S equation is an efficient and practical difference scheme. 
Table 3 The price of a European call option $(\theta=1, \alpha=1,2 / 3,1 / 2,1 / 3)$

\begin{tabular}{llllll}
\hline Time (T/month) & $\mathbf{3}$ & $\mathbf{6}$ & $\mathbf{9}$ & $\mathbf{1 2}$ & CPU time (s) \\
\hline$\alpha=1$ & 44.9980 & 45.2358 & 45.4745 & 45.7157 & 0.45 \\
$\alpha=2 / 3$ & 45.1751 & 45.6286 & 46.1718 & 47.1348 & 0.43 \\
$\alpha=1 / 2$ & 45.4855 & 46.3806 & 47.6890 & 51.5890 & 0.45 \\
$\alpha=1 / 3$ & 45.6278 & 46.8333 & 49.0078 & 59.5237 & 0.46 \\
\hline
\end{tabular}

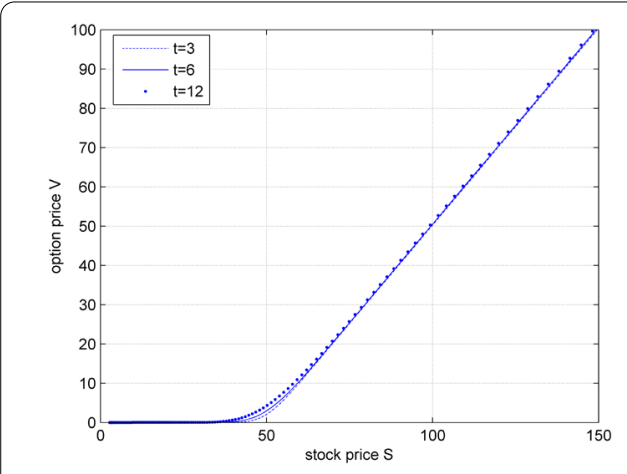

(a) $\alpha=1$

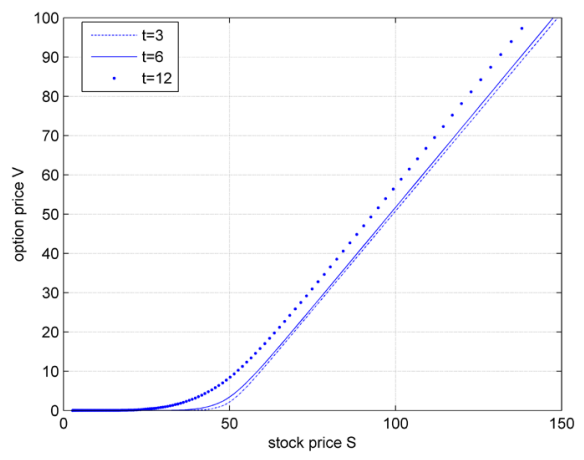

(c) $\alpha=1 / 2$

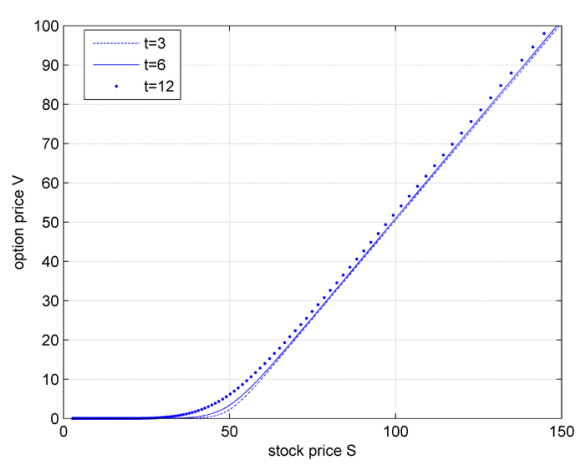

(b) $\alpha=2 / 3$

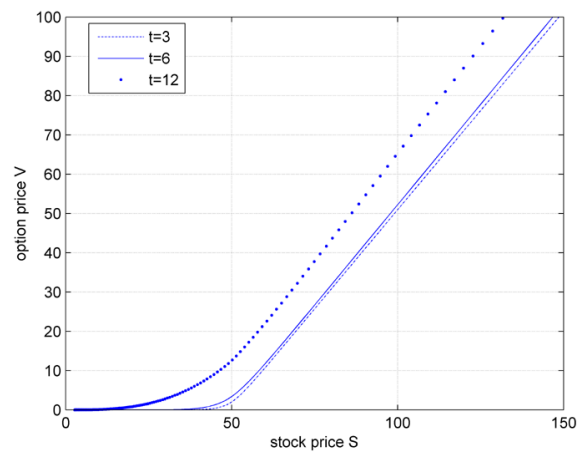

(d) $\alpha=1 / 3$

Figure 3 The price of a European call option $(\theta=1, \alpha=1,2 / 3,1 / 2,1 / 3)$.

In order to further examine the effectiveness of the time-space fractional B-S option pricing modeling and the feasibility of the universal difference scheme method for solving the time-space fractional B-S equation, we will compute the numerical solutions with the condition of case III and IV. Specific plan is as follows: select an unconditional stability implicit scheme $(\theta=1)$ and the Crank-Nicolson scheme $(\theta=1 / 2)$, study of the effect of $\alpha$ value for option price, the value of $\alpha$ selected $2 / 3,1 / 2,1 / 3$, respectively.

Case III:

$$
\theta=1, \quad \alpha=1,2 / 3,1 / 2,1 / 3, \quad M=200, \quad N=120, \quad k=0.0083, \quad h=0.0235 .
$$

We get the results in Table 3 and Figure 3.

Case IV:

$$
\theta=1 / 2, \quad \alpha=1,2 / 3,1 / 2,1 / 3, \quad M=200, \quad N=120, \quad k=0.0083, \quad h=0.0235 .
$$

We get the results in Table 4 and Figure 4 . 
Table 4 The price of a European call option $(\theta=1 / 2, \alpha=1,2 / 3,1 / 2,1 / 3)$

\begin{tabular}{llllll}
\hline Time (T/month) & $\mathbf{3}$ & $\mathbf{6}$ & $\mathbf{9}$ & $\mathbf{1 2}$ & CPU time (s) \\
\hline$\alpha=1$ & 44.9940 & 45.2318 & 45.4705 & 45.7114 & 0.33 \\
$\alpha=2 / 3$ & 45.3411 & 46.0406 & 46.9641 & 49.0869 & 0.42 \\
$\alpha=1 / 2$ & 45.2673 & 45.9415 & 47.0145 & 51.3765 & 0.40 \\
$\alpha=1 / 3$ & 45.1913 & 45.8369 & 47.1096 & 55.8129 & 0.37 \\
\hline
\end{tabular}

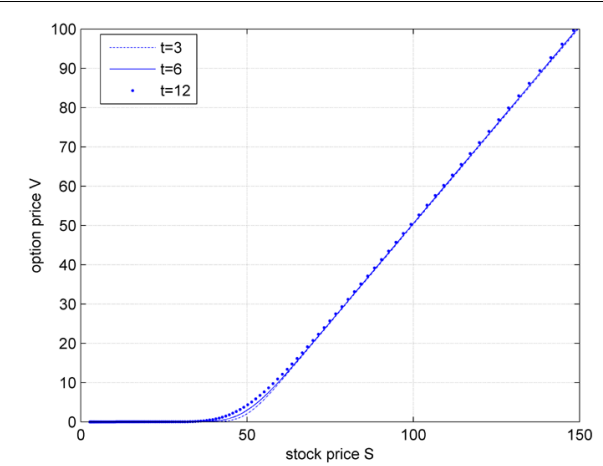

(a) $\alpha=1$

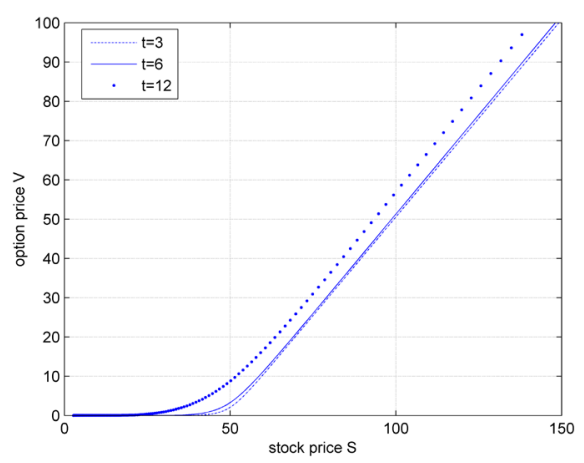

(c) $\alpha=1 / 2$

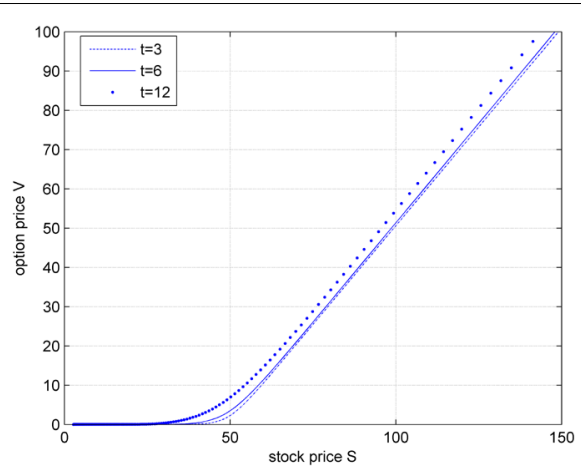

(b) $\alpha=2 / 3$

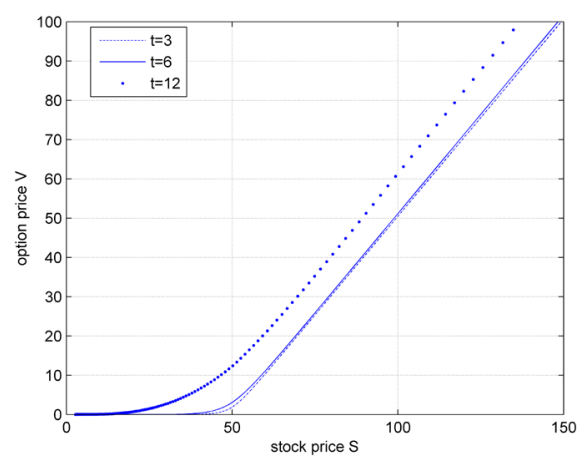

(d) $\alpha=1 / 3$

Figure 4 The price of a European call option $(\theta=1 / 2, \alpha=1,2 / 3,1 / 2,1 / 3)$.

From Figures 3 and 4, we can see that the visible shapes and the trend of the time-space fractional B-S equation are similar to the classical European call option pricing model based on the standard B-S equation $(\alpha=1)$, which illustrates the essential characteristics of the European call options. Therefore, they illustrate that the time-space fractional B-S option pricing equation is effective and the universal difference scheme (6) is feasible for solving the time-space fractional B-S equation.

According to the numerical results in Tables 3, 4 and Figures 3 and 4, when $\frac{1}{2} \leq \alpha<1$ for the time-space fractional B-S equation, the results of the time-space fractional B-S equation are better than the standard B-S equation. The option price of the standard B-S equation $(\alpha=1)$ is small, lower than for the actual financial market (see [24]). It affirms that the time-space fractional B-S equation is more consistent with the actual financial market.

When $0<\alpha<\frac{1}{2}$, the influence of the option price is larger for the time-space fractional B-S equation, that is to say, the option price of 12 months is higher than the standard B-S 
equation. In order to meet the actual financial market, the parameter of the time-space B-S equation should be selected properly according to the actual data.

\section{Conclusions}

In this work, the universal difference method is employed to solve the time-space fractional B-S equation with the boundary conditions satisfied by standard European call options. Theoretical analysis demonstrates that the universal difference method satisfies conditional stability and convergence. Numerical experiments are well in agreement with theoretical analysis. All the results illustrate that the time-space fractional B-S equation is effective and the universal difference scheme is feasible to solve the time-space fractional B-S equation.

\section{Competing interests}

The authors declare that there is no conflict of interests regarding the publication of this paper.

\section{Authors' contributions}

All authors contributed equally and significantly in writing this article. All authors read and approved the final manuscript.

\section{Acknowledgements}

This work is sponsored by the project National Science Foundation of China (No. 11371135), the Fundamental Research Funds for the Central Universities (No. 13QN30).

Received: 4 November 2015 Accepted: 24 February 2016 Published online: 08 March 2016

\section{References}

1. Kwok, Y: Mathematical Models of Financial Derivatives, 2nd edn. Springer, Berlin (2008)

2. Jiang, LS, Xu, CL, et al.: Mathematical Model and Case Analysis of the Pricing of Financial Derivatives. Higher Education Press, Beijing (2008) (in Chinese)

3. Wyss, W: The fractional Black-Scholes equations. Fract. Calc. Appl. Anal. 3(1), 51-61 (2000)

4. Jumarie, G: Stock exchange fractional dynamics defined as fractional exponential growth driven by Gaussian white noise. Application to fractional Black-Scholes equations. Insur. Math. Econ. 42(1), 271-287 (2008)

5. Jumarie, G: Derivation and solutions of some fractional Black-Scholes equations in coarse-grained space and time. Application to Merton's optimal portfolio. Comput. Math. Appl. 59(3), 1142-1164 (2010)

6. Cartea, A, del-Castillo-Negrete, D: Fractional diffusion models of option prices in markets with jumps. Phys. A, Stat. Mech. Appl. 374(2), 749-763 (2007)

7. Zeng, CB, Chen, YQ, Yang, QG: Almost sure and moment stability properties of fractional order Black-Scholes model. Fract. Calc. Appl. Anal. 16(2), 317-331 (2013)

8. Meng, L, Wang, M: Comparison of Black-Scholes formula with fractional Black-Scholes formula in the foreign exchange option market with changing volatility. Asia-Pac. Financ. Mark. 17(2), 99-111 (2010)

9. Barles, G, Soner, HM: Option pricing with transaction costs and a nonlinear Black-Scholes equation. Finance Stoch. 2(4), 369-397 (1998)

10. Kou, SG: A jump-diffusion model for option pricing. Manag. Sci. 48(8), 1086-1101 (2002)

11. Diethelm, K: The Analysis of Fraction Differential Equations. Springer, Berlin (2010)

12. Guo, BL, Pu, XK, Huang, FH: Fractional Partial Differential Equations and Their Numerical Solutions. Science Press, Beijing (2011) (in Chinese)

13. Sun, ZZ, Gao, GH: Finite Difference Method for Fractional Differential Equations. Science Press, Beijing (2015) (in Chinese)

14. Tan, PY, Zhang, XD: A numerical method for the space-time fractional convection-diffusion equation. Math. Numer. Sin. 30(3), 305-310 (2008) (in Chinese)

15. Langlands, TAM, Henry, BI: The accuracy and stability of an implicit solution method for the fractional diffusion equation. J. Comput. Phys. 205, 719-736 (2005)

16. Tadjeran, C, Meerschaert, MM, Scheffler, HP: A second-order accurate numerical approximation for the fraction diffusion equation. J. Comput. Phys. 213(1), 205-213 (2006)

17. Liu, F, Zhuang, P, Anh, V, Turner, I, Burrage, K: Stability and convergence of the difference methods for the space-time fractional advection-diffusion equation. Appl. Math. Comput. 191(1), 12-20 (2007)

18. Chen, CM, Liu, FW, Kevin, B: Finite difference methods and a Fourier analysis for the fractional reaction-subdiffusion equation. Appl. Math. Comput. 198(2), 754-769 (2008)

19. Kumar, S, Yildirim, A, et al.: Analytical solution of fractional Black-Scholes European option pricing equation by using Laplace transform. J. Fract. Calc. Appl. 2(8), 1-9 (2012)

20. Kumar, S, Kumar, D, Singh, J: Numerical computation of fractional Black-Scholes equation arising in financial market. Egypt. J. Basic Appl. Sci. 1(3-4), 177-183 (2014)

21. Ghandehari, MAM, Ranjbar, M: European option pricing of fractional Black-Scholes model with new Lagrange multipliers. Comput. Methods Differ. Equ. 2(1), 1-10 (2014)

22. Song, LN, Wang, WG: Solution of the fractional Black-Scholes option pricing model by finite difference method. Abstr. Appl. Anal. 2013, Article ID 194286 (2013) 
23. Yang, XZ, Zhang, X, Wu, LF: A kind of efficient difference method for time-fractional option pricing model. Appl. Math. J. Chin. Univ. Ser. A 30(2), 234-244 (2015) (in Chinese)

24. Carr, P, Wu, LR: Time-changed Levy processes and option pricing. J. Financ. Econ. 71(1), 113-141 (2004)

Submit your manuscript to a SpringerOpen ${ }^{\circ}$ journal and benefit from:

- Convenient online submission

Rigorous peer review

- Immediate publication on acceptance

- Open access: articles freely available online

- High visibility within the field

- Retaining the copyright to your article

Submit your next manuscript at $\boldsymbol{s p r i n g e r o p e n . c o m ~}$ 